\title{
Effects of an 8-week meditation program on mood and anxiety in patients with memory loss.
}

\author{
Aleezé Sattar Moss \\ Jefferson-Myrna Brind Center of Integrative Medicine \\ Nancy Wintering \\ Jefferson-Myrna Brind Center of Integrative Medicine \\ Hannah Roggenkamp \\ Jefferson-Myrna Brind Center of Integrative Medicine \\ Dharma Singh Khalsa \\ Alzheimer Research and Prevention Foundation, Tucson, AZ

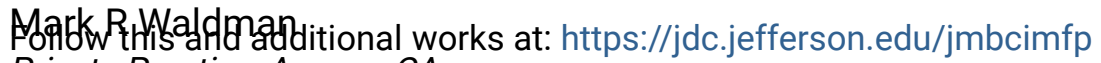 \\ Private Practice, Agoura, CA \\ Part of the Alternative and Complementary Medicine Commons \\ Let us know how access to this document benefits you
}

\section{Recommended Citation}

Moss, Aleezé Sattar; Wintering, Nancy; Roggenkamp, Hannah; Khalsa, Dharma Singh; Waldman, Mark R; Monti, Daniel; and Newberg, Andrew B, "Effects of an 8-week meditation program on mood and anxiety in patients with memory loss." (2012). Marcus Institute of Integrative Health Faculty Papers. Paper 6.

https://jdc.jefferson.edu/jmbcimfp/6

This Article is brought to you for free and open access by the Jefferson Digital Commons. The Jefferson Digital Commons is a service of Thomas Jefferson University's Center for Teaching and Learning (CTL). The Commons is a showcase for Jefferson books and journals, peer-reviewed scholarly publications, unique historical collections from the University archives, and teaching tools. The Jefferson Digital Commons allows researchers and interested readers anywhere in the world to learn about and keep up to date with Jefferson scholarship. This article has been accepted for inclusion in Marcus Institute of Integrative Health Faculty Papers by an authorized administrator of the Jefferson Digital Commons. For more information, please contact: JeffersonDigitalCommons@jefferson.edu. 


\section{Authors}

Aleezé Sattar Moss, Nancy Wintering, Hannah Roggenkamp, Dharma Singh Khalsa, Mark R Waldman, Daniel Monti, and Andrew B Newberg 


\title{
Effects of an 8-Week Meditation Program on Mood and Anxiety in Patients with Memory Loss
}

\author{
Aleezé Sattar Moss, PhD, Nancy Wintering, MSW, Hannah Roggenkamp, BA, Dharma Singh Khalsa, MD, ${ }^{2}$ \\ Mark R. Waldman, BA, ${ }^{3}$ Daniel Monti, MD, and Andrew B. Newberg, MD ${ }^{1}$
}

\begin{abstract}
Background: This study assesses changes in mood and anxiety in a cohort of subjects with memory loss who participated in an 8-week Kirtan Kriya meditation program. Perceived spirituality also was assessed. Previous reports from this cohort showed changes in cognitive function and cerebral blood flow (CBF). The purpose of this analysis was to assess outcome measures of mood and affect, and also spirituality, and to determine whether or not results correlated with changes in CBF.

Methods: Fifteen (15) subjects (mean age $62 \pm 7$ years) with memory problems were enrolled in an 8-week meditation program. Before and after the 8-week meditation, subjects were given a battery of neuropsychologic tests as well as measures of mood, anxiety, and spirituality. In addition, they underwent single photon emission computed tomography scans before and after the program. A region-of-interest template obtained counts in several brain structures that could also be compared to the results from the affect and spirituality measures.

Results: The meditation training program resulted in notable improvement trends in mood, anxiety, tension, and fatigue, with some parameters reaching statistical significance. All major trends correlated with changes in CBF. There were nonsignificant trends in spirituality scores that did not correlate with changes in CBF.

Conclusions: An 8-week, 12 minute a day meditation program in patients with memory loss was associated with positive changes in mood, anxiety, and other neuropsychologic parameters, and these changes correlated with changes in CBF. A larger-scale study is needed to confirm these findings and better elucidate mechanisms of change.
\end{abstract}

\section{Introduction}

$\mathbf{M}$ EMORY LOSS AND AGE-ASSOCIATED cognitive problems are significant issues for an aging American population. According to the census data, $13 \%$ of the U.S. population aged 65 and $25 \%-50 \%$ of those aged 85 or older have symptoms of Alzheimer disease (AD). ${ }^{1,2}$ Some of the early symptoms of $\mathrm{AD}$ include memory loss, confusion regarding time and place, problems with words in speaking or writing, as well as changes in mood and personality. Somewhere between $10 \%$ and $20 \%$ of people aged 65 and older and $29 \%$ of those 85 or older have mild cognitive impairment $(\mathrm{MCI})^{3}$ that may develop into AD. People with MCI have problems with memory, language, or another essential cognitive function that is noticeable to others but not severe enough to interfere with daily life. Age-associated cognitive impairment can be accompanied by depression and changes in mood, 4,5 and the data suggest that mood disorders can aggravate the processes of cognitive decline. ${ }^{6}$ The effect of aging and AD on spirituality is less clear, although individuals with early AD have frequently turned to spirituality as an important coping mechanism. ${ }^{7}$ In fact, higher levels of spirituality have been associated with a slower rate of cognitive decline in patients with $\mathrm{AD} .^{8}$

There are not many treatment options for age-associated memory loss and cognitive impairment, especially with associated mood-related problems. While some medication and vaccine trials are under way, a cost-effective nonpharmacological approach without side-effects that could be easily implemented would be extremely useful in the treatment of early cognitive impairment and memory loss and associated mood disorders in the elderly. Initial studies indicate that meditation can be helpful for emotional regulation as well as cognitive improvement., $10,11,12,13,14,15$ While there are many different forms of meditation, a common element in all meditative techniques is the regulation of

\footnotetext{
${ }^{1}$ Jefferson-Myrna Brind Center of Integrative Medicine, Philadelphia, PA.

${ }^{2}$ Alzheimer Research and Prevention Foundation, Tucson, AZ.

${ }^{3}$ Private Practice, Agoura, CA.
} 
attention and emotion. Recent neuroimaging studies have demonstrated neurological correlates of meditation, highlighting brain regions that regulate attentional control and affect. $^{16,17,18,19,20,21}$

This study was conducted to investigate the potential effects of a specific form of meditation called Kirtan Kriya (KK) on cerebral blood flow (CBF), cognitive effects, and mood in elderly subjects with actual memory loss. Functional brain imaging with single photon emission computed tomography (SPECT) was used to measure potential changes in CBF. Additionally, neurocognitive tests were used to evaluate changes in cognitive function and memory, and self-reported measures were used to assess mood and feelings of spirituality. Previously reported findings from this study showed that this simple 8-week meditation program resulted in significant increases $(p<0.05)$ in baseline CBF ratios in the prefrontal, superior frontal, and superior parietal cortices as well as improvements in neuropsychologic tests of verbal fluency, Trails B, and logical memory. ${ }^{22}$ The primary purpose of this article is to report on the effects of performing daily KK meditation for 8 weeks on mood states and feelings of spirituality and to correlate potential effects with changes in $\mathrm{CBF}$, and improvements in memory.

KK meditation employs a simple technique that involves the repetition of four sounds: SA TA NA MA. While the person vocalizes these sounds, they sequentially touch their thumb to their index finger, middle finger, fourth finger, and then fifth finger. This is performed out loud for 2 minutes, in a whisper for 2 minutes, in silence for 4 minutes, followed by in a whisper for 2 more minutes, and finally out loud for the final 2 minutes. The total time is 12 minutes. Since this is a simple and quick practice, it has the potential to be a very practical and low-cost measure to help improve memory and mood in the elderly. This also distinguishes KK practice from a number of other meditation practices that require extended class sessions and long meditation practices that may not be practical in an older population. While KK originates in the Kundalini yoga tradition, for the purposes of this research it was taught in a secular manner.

Subjects were studied using SPECT imaging to assess changes in CBF at baseline and after practicing the meditation on a daily basis for 8 weeks. Subjects also completed subjective measures of anxiety, depression, and spirituality. It was hypothesized that several brain structures would be particularly affected by the KK meditation program that could be correlated with changes in mood and possibly feelings of spirituality. Studies have shown that attentionfocusing practices such as meditation activate the attentional network in the brain, which includes frontal lobe structures as well as the anterior cingulate cortex. ${ }^{23,24,25}$ These areas also help mediate emotional responses and have also been observed to be related to a variety of spiritual measures.

\section{Materials and Methods}

\section{Subjects and imaging acquisition}

Fifteen (15) subjects complaining of memory problems ranging from mild age associated memory impairment $(N=7)$, to mild cognitive impairment (MCI) $(N=5)$, to moderate impairment with a diagnosis of $\mathrm{AD}(N=3)$ were recruited into the study. The Mini-Mental Status Examination scores (MMSE) ranged from 16 to 30 with a mean of
$28 \pm 3$. There were 6 men and 9 women with ages ranging from 52 to 77 years, with a mean age of $62 \pm 7$ years.

None of the subjects had significant experience with meditation or yoga. Subjects were studied on their first KK training day and then again after an 8-week self-directed training program. On the first day of the study, a baseline SPECT scan was conducted after the subject rested in the room with their eyes closed and listened to a general informational CD about the effects of meditation practices for approximately 12 minutes. This CD was neutral in its content. This scan was labeled the "pre-program baseline" scan.

Following this pre-program baseline scan, the subject returned to the room for their first meditation session. Subjects initially viewed a 10-minute video on how to perform the Kirtan Kriya meditation. Subjects were not asked to do anything more than perform the task. Thus, there were no additional instructions regarding the state of mind that they should be in, any preparatory exercises, or any mindfulness exercises. At the end of the video, the principal investigator answered any questions and then observed the subjects doing the meditation to make sure that it was done correctly. Subjects were instructed that they would perform the meditation while listening to a meditation CD that guided them through the entire practice. The CD contains an individual performing the meditation practice in its intended manner with some light background music to aid in the performance of the meditation. The subjects were then asked to perform the meditation for 12 minutes the first time. The subject was then scanned for 30 minutes using the same imaging parameters as for the baseline study. This scan was labeled the "pre-program meditation" scan.

Subjects were sent home with the meditation CD so that they could practice it at home. They were instructed to perform the practice every day for 8 weeks. Subjects completed a log to record when they performed the meditation practice and their subjective experience of the practice and its effects. No subjects were excluded from the study based on their responses, but additional statistical analyses were done to determine whether there was any effect related to the amount of meditation performed. Upon completion of the 8-week meditation training program, subjects returned to undergo a second imaging day essentially identical to the first. They received a "post-program baseline" scan after listening again to an informational CD. After the baseline scan, the subjects then performed the meditation for the final time, after which they underwent a "post-program meditation" scan. The same order was maintained in the pre- and post-program imaging studies so that the effect of doing the meditation would not interfere with the baseline scans.

A comparison group was recruited, after the initial cohort, in which KK meditation was replaced with "music listening." Five (5) subjects, 2 with MCI and 3 with aging-associated memory impairment (all women with a mean age of $69 \pm 9$ years and a range from 56 to 79 and mean MMSE of 28 \pm 2 ), were asked to simply listen to two Mozart violin concertos each day for approximately 12 minutes, the same amount of time required for the KK meditation. The subjects were asked to listen to the music and to record their progress in a log book. Subjects underwent the same SPECT imaging procedures as the KK group, with listening to the music replacing listening and performing KK meditation. The music group listened to the same informational CD with neutral content. 
Therefore, the baseline states represented both subject groups listening to the same information, allowing for adequate comparison of these baseline scans. In addition, by listening to music for the same amount of time as the subjects who performed KK meditation, this provided an appropriate comparison for the KK meditation program since subjects would undergo similar types of programs with the exception of not doing the active part of the KK meditation.

Subjects in both groups were also evaluated on the first imaging day with a series of questionnaires including the Speilberger State and Trait Anxiety Inventory, ${ }^{26}$ which measures both the temporary condition of "state anxiety" and the more general condition of "trait anxiety"; and the Profile of Mood States (POMS), which measures identifiable mood or affective states: Tension-Anxiety, Depression-Dejection, Anger-Hostility, Fatigue-Inertia, and Confusion-Bewilderment. ${ }^{27}$ Measures of spiritually related feelings included the Index of Core Spiritual Experiences (INSPIRIT) ${ }^{28}$ the Purpose in Life Scale, ${ }^{29}$ the Mysticism Scale, ${ }^{30}$ and the Quest Scale. ${ }^{31}$ Subjects also completed the Mindful Attention Awareness Scale. ${ }^{32}$ These same tests were repeated at the 8 -week postprogram session. Similarly, a neuropsychologic test battery was performed on the pre- and postprogram scan days and comprising a Category Fluency task in which subjects named as many animals as possible in a 60-second time period, the Wechsler Adult Intelligence Scale Digit Symbol Substitution Test, a Logical Memory task, and Trails A and B. These tests also were selected based upon other studies in which neuropsychologic tests were used to evaluate changes in cognition associated with mental task interventions. ${ }^{33,34}$

\section{Image analysis and statistics}

The images of the pre- and postprogram baseline and meditation scans were reconstructed and resliced, using an oblique reformatting program, according to the anteriorposterior commissure line so that the final two sets were aligned for analysis. All images were de-identified and analyses were performed blinded to the patient or the pre/post state of the brain. A previously validated template methodology consisting of regions of interest (ROI) corresponding to the major cortical and subcortical structures was placed over the baseline scan. ${ }^{35}$ For the purposes of this study, the CBF was examined as measured in only a selected number of ROIs, which was hypothesis driven. The ROIs examined included the inferior frontal, superior frontal, dorsolateral prefrontal, inferior temporal, superior temporal, inferior parietal, superior parietal, as well as the amygdala, caudate, thalamus, and cingulate gyrus since these are areas that have been found to be previously affected during meditation tasks and also because these structures subserve a number of cognitive and affective responses. The location for each ROI was determined based upon magnetic resonance imaging (MRI) anatomy such that they could then be placed directly on functional SPECT scans. Furthermore, each ROI (which fit within each specified region and therefore represents a "punch biopsy" of any given area in order to ensure proper placement and to avoid problems with partial voluming) were placed on the initial scan and then copied directly onto all subsequent scans. This was possible because the images were already resliced into the same planes as described above. The count values for the baseline and meditation scans were obtained by determining the number of counts in each ROI on the meditation scan and normalizing those counts to the whole brain activity. This provides a CBF ratio for each ROI compared to the whole brain. Since two SPECT scans were performed on the same day, the second scan had the decay-corrected counts from the first scan subtracted out prior to analysis. This technique has previously been validated and it was shown that there is a high test-retest correlation with less than $6 \%$ variability. ${ }^{36}$

A percentage change between the meditation and baseline scans (for both the pre- and postprogram sessions) was calculated using the equation:

$$
\% \text { Change }=\frac{(\text { Meditation }- \text { Baseline })}{(\text { Baseline })} \times 100
$$

Measures of affect and spirituality were compared using paired $t$ tests. A linear regression model was used to correlate changes in mood with changes in memory. A limited number of Pearson correlations between changes in neuropsychologic test scores and changes in the pre- and postbaseline CBF were compared for selected regions that are known to be related to such parameters. Thus, the amygdala and caudate nucleus were compared to emotional states of tension and depression and the amygdala, frontal lobe, and temporal lobe CBF were compared to feelings of spirituality. The CBF data analyses for multiple comparisons were corrected using the False Discovery Rate method. ${ }^{37}$

\section{Results}

The characteristics of the KK and music listening groups are not significantly different and are given in Table 1 . Primary results of the SPECT scans were previously reported and showed significant changes between the pre and postprogram scans in $\mathrm{CBF}^{22}$ Structures in the frontal lobe regions and right superior parietal lobe had significantly higher baseline CBF after the 8-week training program and that were associated with improvements in several memory tests.

Regarding the POMS, the KK group showed pre/post improvement in all categories, although significance was only observed in the fatigue subcategory (Table 2). When compared

Table 1. Baseline Characteristics of the Kirtan KriYa (KK) and Music Comparison Groups

\begin{tabular}{lccc}
\hline Baseline characteristics & KK group & Music group & $\mathrm{p}$ \\
\hline Age & $64.0 \pm 8.0$ & $65.0 \pm 9.9$ & N.S. \\
MMSE & $28.1 \pm 0.7$ & $29.0 \pm 1.0$ & N.S. \\
$\begin{array}{l}\text { Category fluency } \\
\quad \text { (animals) }\end{array}$ & $21.1 \pm 7.9$ & $21.5 \pm 5.0$ & N.S. \\
Trails B & $105.5 \pm 52.8$ & $132.5 \pm 58.6$ & N.S. \\
Logical memory & $10.6 \pm 5.2$ & $12.3 \pm 6.5$ & N.S. \\
$\quad$ delayed & & & \\
POMS & $52.2 \pm 12.9$ & $47.5 \pm 17.2$ & N.S. \\
Tension & $8.9 \pm 5.4$ & $9.0 \pm 5.0$ & N.S. \\
Depression & $6.6 \pm 4.8$ & $4.8 \pm 4.6$ & N.S. \\
Anger & $4.7 \pm 3.4$ & $2.8 \pm 3.0$ & N.S. \\
Fatigue & $9.2 \pm 9.0$ & $6.3 \pm 4.6$ & N.S. \\
Confusion & $8.4 \pm 5.4$ & $9.5 \pm 4.5$ & N.S. \\
\hline
\end{tabular}

MMSE, Mini-Mental Status Examination; POMS, Profile of Mood States; N.S., not significant. 
Table 2. Profile of Mood States (POMS)

For Both the KIRTAN KRIYA (KK) Group and Music Group Pre- and Post the 8-Week Program

\begin{tabular}{lcccc}
\hline POMS scores & Pre & Post & \% Change & $\mathrm{p}$ \\
\hline KK group & & & & \\
Tension & $8.9 \pm 5.4$ & $6.6 \pm 5.2$ & -26 & 0.04 \\
Depression & $6.6 \pm 4.8$ & $5.2 \pm 4.6$ & -18 & 0.20 \\
Anger & $4.7 \pm 3.4$ & $4.2 \pm 3.6$ & -10 & 0.29 \\
Fatigue & $9.2 \pm 9.0$ & $4.8 \pm 5.2$ & -48 & 0.02 \\
Confusion & $8.4 \pm 5.4$ & $5.8 \pm 4.4$ & -31 & 0.11 \\
Music group & & & & \\
Tension & $9.0 \pm 5.0$ & $16.5 .0 \pm 13.5$ & +83 & 0.13 \\
Depression & $4.8 \pm 4.6$ & $5.3 \pm 3.5$ & +10 & 0.35 \\
Anger & $2.8 \pm 3.0$ & $4.3 \pm 4.3$ & +54 & 0.19 \\
Fatigue & $6.3 \pm 4.6$ & $12.3 . \pm 5.7$ & +95 & 0.01 \\
Confusion & $9.5 \pm 4.5$ & $10.0 \pm 2.6$ & +5 & 0.10 \\
\hline
\end{tabular}

to the music control group, the POMS scores were significantly improved in the KK group in both the tension and fatigue categories $(p<0.05)$. The other POMS scores were better in the KK group, but did not reach significance. There was significant pre/post improvement in the Spielberger state anxiety score for the KK group (pre 35.2 \pm 7.9 and post $30.1 \pm 7.7, p=0.01$ ), but no significant change in trait anxiety score.

There were no significant changes in spirituality scores over the 8- week period. Some trends were noted that approached significance including a slight decrease in the INSPIRIT and Mysticism scores, and a slight increase in Purpose in Life. There was also a trend toward an increase in mindfulness scores (pre score mean was $3.8 \pm 0.5$ and post score mean was $4.1 \pm 0.6, p=0.06$ ). None of the spiritual measures were found to correlate with measures of CBF.

In the KK group, there were several significant correlations observed between the change in baseline CBF ratios and the change in results for the POMS scores (Table 3). Specifically, areas such as the amygdala and caudate correlated with depression scores while the prefrontal cortex, inferior frontal lobe, parietal region, and cingulate cortex correlated with feelings of tension.

Interestingly, there were two significant correlations between the change in POMS scores (confusion and depression) and change in verbal memory as assessed by category fluency memory test $(R=0.63, p=0.005 ; R=0.59, p=0.01$, re-

Table 3. Correlation Between Change in Profile of Mood States (POMS) Scores and Change in Baseline Cerebral Blood Flow (the Correlations that Are Given were Significant After Correction FOR Multiple COMPARISONS)

\begin{tabular}{llcc}
\hline POMS category & \multicolumn{1}{c}{ Structure } & $\mathrm{R}$ & $\mathrm{p}$ \\
\hline Tension & R Prefrontal cortex & 0.73 & 0.016 \\
Tension & R Inferior frontal & 0.77 & 0.008 \\
Tension & R Parietal & 0.80 & 0.005 \\
Tension & R Cingulate & 0.65 & 0.021 \\
Depression & L Superior frontal & 0.83 & 0.002 \\
Depression & R Amygdala & 0.67 & 0.022 \\
Depression & R Caudate & 0.65 & 0.021 \\
Anger & L Superior temporal & 0.86 & 0.002 \\
Fatigue & R Cingulate & 0.73 & 0.017 \\
\hline
\end{tabular}

spectively). This suggests that the improvement in feelings of confusion and depression was related to the cognitive improvement. No other scores of affect and cognition were significantly correlated.

It should be noted that log books and exit interviews with the subjects revealed that the subjects in general found the meditation practice enjoyable and beneficial. The subjects were able to perform the practice a mean of $75 \%$ of the days that they were in the study. Most subjects reported that they subjectively perceived improvements in their mental wellbeing after the 8-week program. However, it was not possible to find any correlation between the amount of practice reported and changes in $\mathrm{CBF}$ or affect scores.

\section{Discussion}

In this study, several neuropsychologic and neurophysiologic changes were observed, and the results suggest that the intervention may be useful for some of the mood and quality-oflife issues that often are associated with cognitive decline. These observations are consistent with a growing literature base of other meditation practices such as mindfulness meditation, which has been shown to reduce anxiety, depression, and psychologic distress in a variety of populations $38,39,40,41,42,43$ including those with chronic medical conditions. ${ }^{44,45,46,47,48,49,50}$ Likewise, a pilot study of mantra meditation with veterans found that a 5-week program significantly reduced symptoms of stress and anxiety and improved feelings of spirituality and well-being. ${ }^{51}$ Mantra meditation is similar to KK practice in that it involves the repetition of certain words or phrases either out loud or silently. ${ }^{52}$

Neuroimaging studies have started to illustrate the neurobiologic correlates of meditation, highlighting brain regions that regulate attention control and affect. While studies utilizing positron emission tomography, SPECT, and functional MRI (fMRI) have all demonstrated specific changes in cortical and subcortical structures when subjects were actively meditating, $53,54,55,56,57,58$ the evidence suggests that different meditation practices lead to different neuropsychologic outcomes. ${ }^{17,18,20,22,59,60,61}$

The preliminary data from the present study show a correlation between changes in the baseline CBF in the right amygdala and right caudate with a reduction in the feeling of depression. A previous fMRI study by Lutz et al. (2008) reported increased $\mathrm{CBF}$ in the amygdala associated with improved emotional processing. ${ }^{62}$ Other meditation studies, including the Cohen et al. prior study on the effects of yoga on $\mathrm{CBF}^{63}$ have reported significant effects in the amygdala, as well as the striatum. ${ }^{17}$ Furthermore, these areas are frequently implicated in depression by a variety of functional neuroimaging studies. ${ }^{64}$ The current data also suggest a correlation between the change in baseline CBF in the right anterior cingulate with a reduction in tension for participants in the KK group. This is consistent with previous studies that have shown the anterior cingulate to be associated with both mood as well as the brain's response to stress. ${ }^{65}$ Also, it was previously reported that the anterior cingulate is activated during meditation practices. ${ }^{66}$ Habituation is potentially a contributing factor to brain changes during the practice of meditation itself; however, it would not explain changes in the baseline brain function that are reported here. There also are correlations between improvements in emotional states 
and memory, but it is important to underscore the small sample size and statistical nonsignificance of many of these observations. Finally, subjects were able to perform the practice a mean of $75 \%$ of the days that they were in the study, and most subjects reported that they subjectively perceived improvements in their mental well-being after the 8 -week program. While it was not possible to find any correlation between the amount of practice reported and changes in CBF or affect scores, a few studies in the literature suggest that greater expertise in meditation results in greater effect. $^{62,67}$ However, larger studies will be better able to assess how the amount and duration of practice correlates with psychologic and cognitive benefits.

The overall findings from this study are an important initial step in understanding potential mediators, moderators, and neurophysiologic correlates of KK practice. Larger, controlled studies with more elaborate neuropsychologic assessments are required to better evaluate the relationship between meditation, memory, and affect.

\section{Acknowledgments}

We would like to thank the Alzheimer's Research and Prevention Foundation in Tucson, Arizona (www .alzheimersprevention.org) for their generous support of this research project.

\section{Disclosure Statement}

No competing financial interests exist.

\section{References}

1. Herbert L, Scherr P, Bienias J, et al. Alzheimer disease in the US population: Prevalence estimates using the 2000 census. Arch Neurol 2003;60:1119-1122.

2. Alzheimer's Association. 2010 Alzheimer's disease facts and figures. Alzheimer's Dement 2010;6:158-194.

3. Lopez OL, Jagust WJ, DeKosky ST, et al. Prevalence and classification of mild cognitive impairment in the Cardiovascular Health Study Cognition Study. Arch Neurol 2003;60:1385-1389.

4. Ownby RL, Crocco E, Acevedo A, John V, Loewenstein D. Depression and risk for Alzheimer disease: systematic review, meta-analysis, and metaregression analysis. Arch Gen Psychiatry 2006;63:530-8.

5. Rinck M, Becker E. Selective memory and memory deficits in depressed inpatients. Depress Anxiety 2003;17:197-206.

6. Gualtieri CT, Johnson LG. Age related cognitive decline in patients with mood disorders. Prog Neuro-Psychopharmacol Biol Psychiatry 2008;32:962-967.

7. Beuscher L, Beck C. A literature review of spirituality in coping with early-stage Alzheimer's disease. J Clin Nurs 2008;17:88-97.

8. Kaufman Y, Anaki D, Binns M, Freedman M. Cognitive decline in Alzheimer disease: Impact of spirituality, religiosity, and QOL. Neurology 2007;68:1509-1514.

9. Horowitz S. Health benefits of meditation: What the newest research shows. Altern Complement Ther 2010;16:223-228.

10. Fortney L, Taylor M. Mediation in medical practice: A review of the evidence and practice. Prim Care 2010;37:81-90.

11. Grossman P, Niemann L, Schmidt S, Walach H. Mindfulness-based stress reduction and health benefits: A metaanalysis. J Psychosom Res 2004;57:35-43.
12. Jain S, Shapiro SL, Swanick S, et al. A randomized controlled trial of mindfulness meditation versus relaxation training: Effects on distress, positive states of mind, rumination, and distraction. Ann Behav Med 2007;33:11-21.

13. Jha A, Stanley E. Examining the protective effects of mindfulness training on working memory capacity and affective experience. Emotion 2010;10:54-64.

14. Lutz A, Slagter H, Rawlings NB, et al. Mental training enhances attentional stability: Neural and behavioral evidence. J Neurosci 2009;29:13418-13427.

15. Xiong GL, Doraiswamy PM. Does meditation enhance cognition and brain plasticity? Ann N Y Acad Sci 2009;1172:63-69.

16. Davidson RJ, Kabat-Zinn J, Schumacher J, et al. Alterations in brain and immune function produced by mindfulness meditation. Psychosom Med 2003;65:564-570.

17. Lazar SW, Bush G, Gollub RL, et al. Functional brain mapping of the relaxation response and meditation. Neuroreport 2000;11:581-585.

18. Lou HC, Kjaer TW, Friberg L, et al. A 15O-H2O PET study of meditation and the resting state of normal consciousness. Hum Brain Map 1999;7:98-105.

19. Herzog H, Lele VR, Kuwert T, et al. Changed pattern of regional glucose metabolism during Yoga meditative relaxation. Neuropsychobiology 1990-1991;23:182-187.

20. Cahn BR, Polich J. Meditation states and traits: EEG, ERP, and neuroimaging studies. Psychol Bull 2006;132:180-211.

21. Lazar SW, Kerr CE, Wasserman RH, et al. Meditation experience is associated with increased cortical thickness. Neuroreport 2005;16:1893-1897.

22. Newberg AB, Wintering $\mathrm{N}$, Khalsa DS, et al. Meditation effects on cognitive function and cerebral blood flow in subjects with memory loss: A preliminary study. J Alzheimers Dis 2010;20:517-526.

23. Frith $\mathrm{CD}$, Friston $\mathrm{K}$, Liddle PF, et al. Willed action and the prefrontal cortex in man: A study with PET. Proc R Soc Lond 1991;244:241-246.

24. Posner MI, Petersen SE. The attention system of the human brain. Ann Rev Neurosci 1990;13:25-42.

25. Pagnoni G, Cekic M. Age effects on gray matter volume and attentional performance in Zen meditation. Neurobiol Aging 2007;28:1623-1627.

26. Spielberger CD, Gorsuch RL, Lushene RE. Manual for the State-Trait Anxiety Inventory. Palo Alto, CA: Consulting Psychologists Press, 1970.

27. McNair DM, Lorr M, Droppleman LF. Manual for the Profile of Mood States. San Diego, CA: EdITS/Educational and Industrial Testing Service, 1992.

28. Kass JD, Friedman R, Lesserman J, et al. Health outcomes and a new index of spiritual experience. J Sci Study Relig 1991;30:203-211.

29. Marsh A, Smith L, Piek J, Saunders B. The purpose in life scale: Psychometric properties for social drinkers and drinkers in alcohol treatment. Educ Psychol Meas 2003;63:859.

30. Hood RW, Ghobani N, Watson PJ, et al. Dimensions of the mysticism scale: Confirming the three-factor structure in the United States and Iran. J Sci Study Relig 2001;40:691-705.

31. Batson CD, Schoenrade P. Measuring religion as Quest: Validity concerns. J Sci Study Relig 1991;30:416-429.

32. Brown KW, Ryan RM. The benefits of being present: Mindfulness and its role in psychological well-being. J Pers Soc Psychol 2003;84:822-848.

33. Talassi E, Guerreschi M, Feriani M, et al. Effectiveness of a cognitive rehabilitation program in mild dementia (MD) and 
mild cognitive impairment (MCI): A case control study. Arch Gerontol Geriatr 2007;44:391-399.

34. Belleville S. Cognitive training for persons with mild cognitive impairment. Int Psychogeriatr 2008;20:57-66.

35. Resnick SM, Karp JS, Tretsky BI, Gur RE. Comparison of anatomically defined versus physiologically based regional localization: Effects on PET-FDG quantitation. J Nucl Med 1993;34:201-208.

36. Newberg AB, Saffer J, Farrar J, et al. Stability of cerebral blood flow measures using a split-dose technique with $99 \mathrm{mTc}-$ exametazime SPECT. Nucl Med Comm 2005;26:475-478.

37. Benjamini Y, Hochberg Y. Controlling the false discovery rate: A practical and powerful approach to multiple testing. J R Stat Soc Ser B (Methodological) 1995;57:289-300.

38. Miller JJ, Fletcher K, Kabat-Zinn J. Three-year follow-up and clinical implications of a mindfulness meditation-based stress reduction intervention in the treatment of anxiety disorders. Gen Hosp Psychiatr 1995;17:192-200.

39. Brown KW, Ryan RM. The benefits of being present: Mindfulness and its role in psychological well-being. J Pers Soc Psychol 2003;84:822-848.

40. Carmody J, Reed G, Kristeller J, Merriam P. Mindfulness, spirituality, and health-related symptoms. J Psychosom Res 2008;64:393-403.

41. Reibel DK, Greeson JM, Brainard GS, Rosenzweig S. Mindfulness-based stress reduction and health-related quality of life in a heterogeneous patient population. Gen Hosp Psychiatry 2001;23:183-192.

42. Toneatto T, Nguyen L. Does mindfulness meditation improve anxiety and mood symptoms? A review of the controlled research. Can J Psychiatry 2007;52:260-266.

43. Goldin PR, Gross JJ. Effects of mindfulness-based stress reduction (MBSR) on emotion regulation in social anxiety disorder. Emotion 2010;10:83-91.

44. Monti DA, Peterson C, Shakin Kunkel EJ, et al. A randomized, controlled trial of mindfulness-based art therapy for women with cancer. Psychooncology 2006;15:363-373.

45. Ledesma D, Kumano H. Mindfulness-based stress reduction and cancer: A meta-analysis. Psychooncology 2008;18:571579 .

46. Witek-Janusek L, Albuquerque K, Chroniak KR, et al. Effect of mindfulness based stress reduction on immune function, quality of life and coping in women newly diagnosed with early stage breast cancer. Brain Behav Immun 2008;969-981.

47. Rosenzweig S, Reibel DK, Greeson JM, et al. Mindfulnessbased stress reduction is associated with improved glycemic control in type 2 diabetes mellitus: A pilot study. Altern Ther Health Med 2007;13:36-38.

48. Rosenzweig S, Greeson J, Reibel D, et al. Mindfulness-based stress reduction for chronic pain conditions: Variation in treatment outcomes and role of home meditation practice. J Psychosom Res 2010;68:29-36.

49. Zautra AJ, Davis MC, Reich JW, et al. Comparison of cognitive behavioral and mindfulness meditation interventions on adaptation to rheumatoid arthritis for patients with and without history of recurrent depression. J Consul Clin Psychol 2008;76:408-421.

50. Zylowska L, Ackerman DL, Yang MH, et al. Mindfulness meditation training in adults and adolescents with ADHD: A feasibility study. J Attention Disord 2008;11:737-746.

51. Bormann JE, Smith TL, Becker S, et al. Efficacy of frequent mantram repetition on stress, quality of life, and spiritual well-being in veterans: A pilot study. J Holist Nurs 2005;23: 395-414.
52. Dillbeck MC, Orme-Johnson DW. Physiological difference between Transcendental Meditation and rest. Am Psychol 1987;42:879-881.

53. Brefczynski-Lewis JA, Lutz A, Schaefer HS, et al. Neural correlates of attentional expertise in long-term meditation practitioners. Proc Natl Acad Sci USA 2007;104:11483-488.

54. Newberg AB, Alavi A, Baime M, et al. The measurement of regional cerebral blood flow during the complex cognitive task of meditation: A preliminary SPECT study. Psychiatry Res Neuroimaging 2001;106:113-122.

55. Luders E, Toga AW, Lepore N, Gaser C. The underlying anatomical correlates of long-term meditation: Larger hippocampal and frontal volumes of gray matter. Neuroimage 2009;45:672-678.

56. Vestergaard-Poulsen $\mathrm{P}$, van Beek M, Skewes J, et al. Longterm meditation is associated with increased gray matter density in the brain stem. Neuroreport 2009;20:170-174.

57. Doraiswamy PM, Xiong GL. Does meditation enhance cognition and brain longevity? Ann N Y Acad Sci 2009; 1172:63-69.

58. Galvin JA, Benson H, Deckro GR, et al. The relaxation response: Reducing stress and improving cognition in healthy aging adults. Complement Ther Clin Pract 2006; 12:186-191.

59. Lutz A, Greischar LL, Rawlings NB, et al. Long-term meditators self-induce high-amplitude gamma synchrony during mental practice. Proc Natl Acad Sci USA 2004;101:1636916373.

60. Davidson RJ, Harrington A. Visions of Compassion: Western Scientists and Tibetan Buddhists Examine Human Nature. New York: Oxford University Press, 2004.

61. Newberg AB, Iversen J. The neural basis of the complex mental task of meditation: Neurotransmitter and neurochemical considerations. Med Hypotheses 2003;61:282-291.

62. Lutz A, Brefczynski-Lewis J, Johnstone T, Davidson RJ. Regulation of the neural circuitry of emotion by compassion meditation: Effects of meditative expertise. PLoS One 2008;3:e1897.

63. Cohen DL, Wintering N, Tolles V, et al. Cerebral blood flow effects of yoga training: Preliminary evaluation of 4 cases. J Altern Complement Med 2009;15:9-14.

64. Rigucci S, Serafini G, Pompili M, et al. Anatomical and functional correlates in major depressive disorder: The contribution of neuroimaging studies. World J Biol Psychiatry 2010;11(2 pt 2):165-180.

65. Dedovic K, D'Aguiar C, Pruessner JC. What stress does to your brain: A review of neuroimaging studies. Can J Psychiatry 2009;54:6-15.

66. Wang DJ, Rao H, Korczykowski M, et al. Cerebral blood flow changes associated with different meditation practices and perceived depth of meditation. Psychiatry Res 2011; 191:60-67.

67. Lutz A, Greischar LL, Perlman DM, Davidson RJ. BOLD signal in insula is differentially related to cardiac function during compassion meditation in experts vs. novices. Neuroimage 2009;47:1038-1046.

Address correspondence to: Andrew B. Newberg, MD Jefferson-Myrna Brind Center of Integrative Medicine 1015 Chestnut Street, Suite 412 Philadelphia, PA 19107

E-mail: Andrew.newberg@jefferson.edu 